\title{
Orientation and spatial frequency effects on linear afterimages*
}

\author{
N.J. WADE \\ Liniversity of Dundec. Dundee DDI HH.N. Scotland
}

\begin{abstract}
Single lines and gratings oricnted vertically or at 45 deg were observed as prolonged afterimages in two experiments. The duration of complete visibility axhibited consistent orientation selectivity for the gratings but not for single lines; vertical gratings were visible for longer than were 45-deg gratings. The results are discussed in relation to the hypothesized spatial frequency channels in the human visual system.
\end{abstract}

Single lines viewed as afterimages do not vary in either their duration of visibility or frequency of fragmentation as a function of orientation (Wade. 1972). This result contrasts with the reported orientation selectivity for lines viewed under steady fixation (Craig \& Lichtenstein. 1953) and during optical stabilization (Schmidt. Fulgham, \& Brown. 1971), as well as a variety of other visual spatial tasks (see Howard \& Templeton, 1966). Moreover, a physiological measure. the averaged cortical potential evoked by oscillating gratings, exhibits orientation selectivity: its amplitude is greater for vertical or horizontal gratings than for those at $45 \mathrm{deg}$ (Campbell \& Maffei, 1970; Maffei \& Campbell, 1970). These last experiments involved gratings rather than single lines, and there is some psychophysical evidence for channels selective to spatial frequency in the human visual system (Blakemore \& Campbell, 1969; Blakemore, Nachmias, \& Sutton, 1970; Pantle \& Sekuler, 1968). This is consonant with the existence of such single-unit analyzers in cat visual cortex (Campbell, Cooper, \& Enroth-Cugell, 1969). It is of interest. therefore, to investigate the possible orientation selectivity of spatially repetitive afterimages.

\section{EXPERIMENT I}

Due to closure effects with afterimages, it is not possible to study any but low spatial frequency gratings, and these consist of approximately square-wave luminance profiles. The present experiment was conducted to investigate the effect of orientation on two afterimages, one a single line and the other a low spatial frequency grating. Each figure was oriented at either $0 \mathrm{deg}$ (vertical) or $45 \mathrm{deg}$.

\footnotetext{
*This research was supported by a grant from the Science Research Council. The assistance of $\mathrm{K}$. Greig in the collection and analysis of data is gratefully acknowledged. I thank Max Coltheart for valuable discussions and J. Cormack for conducting the optical examinations.
}

\begin{abstract}
Method
\section{Apparatus}

Afterimages were generated by the discharge from a Multiblitz Report flashgun, the output of which was $120 \mathrm{~J}$ delivered in 2 msec, according to the manufacturer's specifications. The face of the flashgun was masked apart from the aperture(s) comprising the stimulus pattern, which were located $57 \mathrm{in.} \mathrm{from}$ $S$ 's eyes. Either a single line or four equally spaced lines (4 cycles/deg spatial frequency) were viewed; each line subtended $1 \times 1 / 8$ deg at the eye. The apertures could be rotated appropriately to be oriented at either 0 or $45 \mathrm{deg}$. A luminous circle with a visual angle of 2 deg surrounded the pattern so that the center of the figure could be fixated in the darkened room.

A chinrest and forehead support roughly controlled the S's head position. Directly in front of the seated $S$ were two switches, which activated separate timers and channels of a Rustrak event recorder.
\end{abstract}

\section{Subjects}

Twenty-four Ss, mainly from an introductory course in psychology, participated in the experiment. Those who normally wore corrective lenses did so during the experiment.

\section{Procedure}

Afterimages were generated in S's preferred eye, the other eye being occluded. The $S$ was instructed to depress one switch when the line(s) were visible in their entirety and the other when only part of the line(s) was visible. This latter category included the condition in which less than four of the lines comprising the grating were visible in unitary form. Neither switch was depressed when the figure disappeared completely. A practice trial was given in order to facilitate comprehension of the instructions, and this was followed by $10 \mathrm{~min}$ dark adaptation. Four 1-min experimental trials were given, one for each stimulus figure: a single line at 0 and $45 \mathrm{deg}$ and a grating at 0 and $45 \mathrm{deg}$, the order of which was counterbalanced, with the constraint that the single line and grating pairs be presented successively. The intertrial interval was $5 \mathrm{~min}$, during which $S$ remained in the dark. The afterimages were viewed with the eyes closed.

\section{Results}

The mean durations of visibility and fragmentation frequencies for the line and grating afterimages are given in Table 1 for both the whole and the partial responses. Considering the whole-figure visibility, the vertical afterimages were visible for longer than the 45-deg ones. 
Table 1

Mean Durations (Seconds) and Number of Fragmentations for Afterimages of Single Lines and Gratings Oriented at 0 or $45 \mathrm{Deg}$

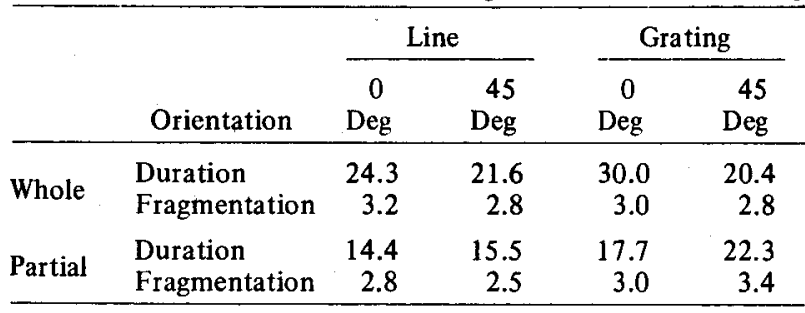

Note-The measures for whole and partial visibility are given separately.

Analysis of the data revealed significant overall differences between the means $[F(3,23)=5.82$, $\mathrm{p}<.01$ ] . Post hoc comparison (Rodger, 1965) indicated that the null hypothesis is rejected for mean differences in excess of $6.1 \mathrm{sec}(p<.05)$. Thus, vertical afterimages of gratings are visible for significantly longer than are those at $45 \mathrm{deg}$, whereas single-line afterimages do not exhibit such orientation selectivity. A further comparison between the means for the lines and the gratings resulted in acceptance of the null hypothesis.

The data for the duration of partial visibility of the afterimages yielded significant differences overall $[F(3,23)=5.84, p<.01]$. Comparisons of the 0 - and 45-deg means for the line and grating afterimages led to acceptance of the null hypothesis, i.e., although the partial visibility of the $45-\mathrm{deg}$ afterimages was longer than that for the vertical, these differences were not significant. The grating stimuli were visible in partial form for significantly longer than the line stimuli $(\mathrm{p}<.05)$.

Analysis of the fragmentation frequencies yielded no significant differences either for the orientation effects or for the line-grating comparisons. This was the case for both the whole and partial data.

The conditional probabilities for the occurrence of a given event contingent upon one of the alternatives are shown in Table 2. The three possible events are the visibility of the complete afterimage (C), some part of it $(\mathrm{P})$, or its total disappearance (D). For the single-line afterimages, the conditional probabilities were very similar for both 0 - and 45-deg orientations, in agreement with the previous study (Wade, 1972). A completely visible line fragmented to a part or disappeared completely with about equal likelihood. Similarly, the frequencies of complete or partial visibility following line disappearance were approximately equal. However, it was more likely that a partially visible line would disappear entirely.

The grating afterimages exhibited more complete-to-partial fragmentation and less partial-to-complete disappearance in comparison to the single lines. This outcome might have been expected because of the broader range of possibilities for the partial category, e.g., the complete visibility of less than the total number of lines, although the number of fragmentations were not significantly greater. There were also some orientation effects on grating fragmentation: the visibility of the whole afterimage fragmented more irequently to a part for the 45-deg grating, and also it was more likely for a part to become visible following a period of afterimage disappearance.

Therefore, spatially repetitive vertical afterimages are visible in unitary form for significantly longer than are those at $45 \mathrm{deg}$, but this orientation difference was not evident for single-line afterimages.

\section{EXPERIMENT II}

The single-line stimuli of the above experiment were equivalent to one bar of the gratings, which contained four such lines. Thus, four times the energy was imparted to the eye for the grating patterns. This experiment investigated the same problem as did the previous one, but a range of stimuli was used so that the energy characteristics remained the same throughout. Stimuli consisting of $1,2,4$, or 8 equally spaced apertures were used, and these were presented in either the vertical or the 45-deg orientation.

\section{Method}

\section{Apparatus}

The apparatus was essentially similar to that used in Experiment I. A Mecablitz 502 flashgun was employed, with output characteristics of $120 \mathrm{~J}$ delivered in $1 \mathrm{msec}$. The face of the flashgun was located $28.5 \mathrm{in}$. from S's eyes, and the apertures were placed directly in front of this. The apertures consisted of either a single line ( $2 \times 1 \mathrm{deg})$ or 2,4 , or 8 equally spaced lines with corresponding spatial frequencies of 1,2 , and 4 cycles/deg; the visual subtenses of the line units of the gratings were $2 \times 1 / 2,2 \times 1 / 4$, and $2 \times 1 / 8 \mathrm{deg}$, respectively. Each of the stimulus patterns could be oriented either vertically or at $45 \mathrm{deg}$. The apertures were surrounded by luminous paint in order to enable fixation of the center of the figure in the darkened room.

\section{Subjects}

Three Ss, well-practised in the observation of afterimages, took part in the experiment. Two Ss (S 1 and $S 2$ ) were

Table 2

Conditional Probabilities for the Occurrence of a Given Event $(N+1)$ Contingent Upon an Alternative $(N)^{*}$

\begin{tabular}{|c|c|c|c|c|}
\hline \multirow[b]{2}{*}{$\mathrm{p}(\mathrm{N}+1 / \mathrm{N})$} & \multicolumn{2}{|c|}{$\begin{array}{c}\text { Line } \\
\text { Orientation }\end{array}$} & \multicolumn{2}{|c|}{$\begin{array}{c}\text { Grating } \\
\text { Orientation }\end{array}$} \\
\hline & $\begin{array}{c}0 \\
\text { Deg }\end{array}$ & $\begin{array}{c}45 \\
\text { Deg }\end{array}$ & $\begin{array}{c}0 \\
\text { Deg }\end{array}$ & $\begin{array}{r}45 \\
\text { Deg }\end{array}$ \\
\hline$p(D / C)$ & .54 & .53 & .42 & .33 \\
\hline $\mathrm{p}(\mathrm{P} / \mathrm{C})$ & .46 & .47 & .58 & .67 \\
\hline $\mathrm{p}(\mathrm{D} / \mathrm{P})$ & .75 & .77 & .54 & .69 \\
\hline$p(C / P)$ & .25 & .23 & .46 & .31 \\
\hline $\mathrm{p}(\mathrm{C} / \mathrm{D})$ & .48 & .55 & .46 & .36 \\
\hline $\mathrm{p}(\mathrm{P} / \mathrm{D})$ & .52 & .45 & .54 & .64 \\
\hline
\end{tabular}

*The three possible events are complete visibility (C). partial visibility $(P)$. and disappearance $(D)$. 
Table 3

Mean Durations (Seconds) and Number of Fragmentations for Single-Line and Grating Afterimages Oriented at 0 and 45 Deg

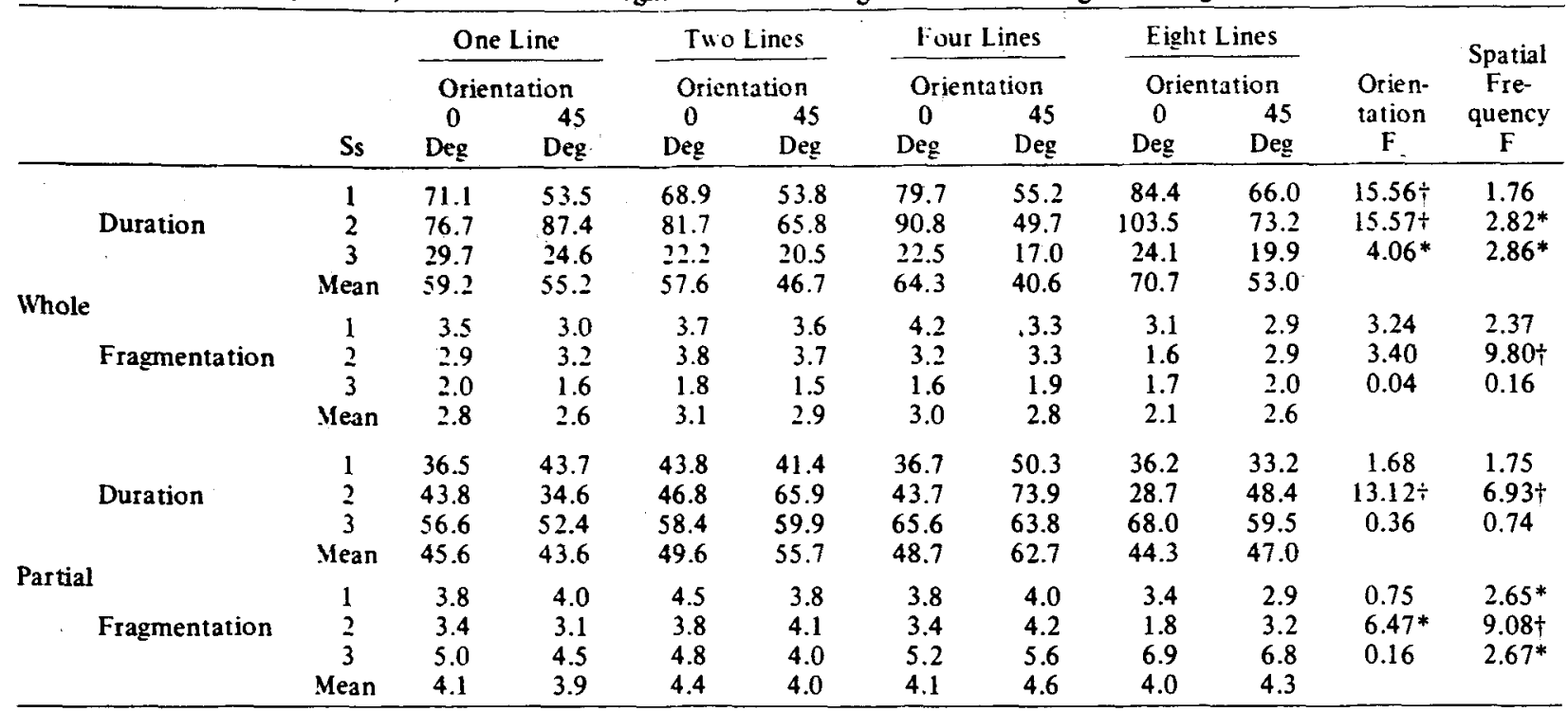

${ }^{*} p<.05 \quad$ tp $<.001$

NOTE-The data for the whole and partial visibility are given separately, as are the F ratios for the orientation and line (spatial frequency/ factors.

emmetropic with the right eye, whereas $S 3$ had slight uncorrected myopia. None of the $S$ s had any evidence of astigmatism.

\section{Procedure}

Observation was with the right eye, and the left eye was occluded. Following dark adaptation for $10 \mathrm{~min}$, an afterimage of one of the stimuli in a given orientation was generated and viewed with the eyes closed for $3 \mathrm{~min}$. One switch was depressed when the afterimage corresponded to the complete stimulus pattern, and the other when only part of it was visible. The intertrial interval was 5 min, during which $S$ was dark adapted. A session consisted of four trials, one for each stimulus pattern in a given orientation, presented in random order. Sixteen sessions were undertaken, eight for each orientation presented in an alternating sequence.

\section{Results}

The data for each $S$ were analyzed separately, and these are presented in averaged form ${ }^{1}$ in Table 3 . The vertical afterimages were visible in their entirety for longer than the 45-deg ones. This effect was more marked with the spatially repetitive patterns. Each $S$ showed a significant orientation effect for whole duration, but there were no consistent differences over spatial frequencies. The vertical gratings were visible for longer than the tilted ones for all Ss, but this orientation selectivity was shown by only two of the three Ss for the single-line afterimages. The interaction between orientation and spatial frequency was significant only for $\mathrm{S} 2$. The whole fragmentation frequencies exhibited no consistent orientation or spatial frequency differences.

Partial durations tended to favor the $45-\mathrm{deg}$ afterimages, although this was significant for only one $\mathrm{S}$.
Again, no consistent effects were found as a function of spatial frequency. The partial fragmentation frequency was insensitive to orientation, but a small significant effect of spatial frequency was evidenced for each $S$. This effect was not, however, in the same direction for all Ss: two Ss gave a reduced partial fragmentation frequency for the higher spatial frequency gratings, whereas the reverse was shown for the other $S$.

The conditional probabilities are given in Table 4 . While there were some differences over Ss, similar patterns of probabilities were produced and the data for the three Ss were pooled. In general, complete visibility of the afterimage was likely to be followed by a fragment, as was complete disappearance. Partial visibility was followed with approximately equal frequency by complete visibility or disappearance. There was a slight tendency for the 45-deg figures to show more fragmentation from complete-to-partial visibility than did the 0-deg afterimages, in correspondence to

Table 4

Conditional Probabilities for the 0 - and $45-\mathrm{Deg}$ Line and Grating Afterimages

\begin{tabular}{|c|c|c|c|c|c|c|c|c|}
\hline \multirow[b]{3}{*}{$\mathrm{p}(\mathrm{N}+1 / \mathrm{N})$} & \multirow{2}{*}{\multicolumn{2}{|c|}{$\frac{\text { One Line }}{\begin{array}{c}\text { Orientation } \\
\text { (Deg) }\end{array}}$}} & \multirow{2}{*}{\multicolumn{2}{|c|}{$\frac{\text { Two Lines }}{\begin{array}{c}\text { Orientation } \\
\text { (Deg) }\end{array}}$}} & \multirow{2}{*}{\multicolumn{2}{|c|}{$\frac{\text { Four Lines }}{\begin{array}{c}\text { Orientation } \\
\text { (Deg) }\end{array}}$}} & \multirow{2}{*}{\multicolumn{2}{|c|}{$\frac{\text { Eight Lines }}{\begin{array}{c}\text { Orientation } \\
\text { (Deg) }\end{array}}$}} \\
\hline & & & & & & & & \\
\hline & 0 & 45 & 0 & 45 & 0 & 45 & 0 & 45 \\
\hline$(D / C)$ & .39 & .31 & .26 & .22 & .34 & .18 & .28 & .28 \\
\hline$(\mathrm{P} / \mathrm{C})$ & .61 & .69 & .74 & .78 & .6 & .82 & .72 & .72 \\
\hline$(\mathrm{D} / \mathrm{P})$ & .55 & .55 & .51 & .50 & .51 & .56 & .57 & .56 \\
\hline$p(C / P)$ & .45 & .45 & .49 & .50 & .49 & .44 & .43 & .44 \\
\hline $\mathrm{p}(\mathrm{C} / \mathrm{D})$ & .28 & .23 & .25 & .26 & .25 & .21 & .20 & .31 \\
\hline $\mathrm{p}(\mathrm{P} / \mathrm{D})$ & .72 & .77 & .75 & .74 & .75 & .79 & .80 & .69 \\
\hline
\end{tabular}


Experiment 1. Otherwise, the difference in the pattern of fragmentations between orientations was slight. There was a higher probability of complete-to-partial fragmentation than in Experiment $I$. This may have been due to the larger angular subtense of the long dimension of the lines in this experiment $(2 \mathrm{deg})$ relative to the previous one (1 deg).

Thus, the results of this experiment provide general support for those of the first one: the duration of unitary visibility favors vertical gratings in a manner not evident consistently for single-line afterimages. This was the case when the energy characteristics of the primary stimulus remained constant.

\section{DISCUSSION}

The results of both experiments support those of the previous study (Wade, 1972) in yielding no evidence of consistent duration differences for single-line afterimages oriented at 0 and $45 \mathrm{deg}$. This was the case for line afterimages subtending widely differing visual angles $(1 \mathrm{x}$ $1 / 8 \mathrm{deg}$ and $2 \times 1 / 2 \mathrm{deg}$ ). Contrariwise, afterimages of gratings do exhibit orientation selectivity, with verticals persisting for longer in unitary form than those at $45 \mathrm{deg}$.

Evans (1967) has suggested that the mode of line afterimage fragmentation reflects properties of receptive fields in the human visual system. The presence of linear analyzers in cat was enlisted in support of this hypothesis. Weisstein (1969) has drawn attention to certain critical difficulties of this approach. More moderately, Atkinson (1972) has postulated that the pattern of afterimage disappearances represents the operation of "functional units" in the human visual system. Both Evans and Atkinson estimated the size of the units from the intersection of the unitary and partial disappearance durations in a $60-\mathrm{sec}$ observation interval, ${ }^{2}$ although their separate estimates were not in close correspondence. This line of reasoning may be applied to the present data (specifically that of Experiment I, which used the same observation period as above), with the added assumption that there exist units responsive to specific spatial frequencies. Consequently, it would appear that vertical gratings have smaller functional units than do 45-deg gratings, as the whole/partial disappearance duration ratio is smaller for the former. This speculation is consonant with the finding that the cortical potential evoked by a vertical grating is larger in amplitude than that evoked by a 45-deg grating of the same visual subtense (Campbell \& Maffei, 1970; Maffei \& Campbell, 1970). That is, more functional units may have been stimulated by the vertical grating. This correspondence also suggests that the origin of the pattern of afterimage degeneration is at the level of the cortex.

The basis of the difference in orientation effects between single lines and gratings may reside in the complexity, in Fourier terms. of the former. Recent applications of Fourier analysis to human perception of visual spatial patterns (Campbell \& Robson, 1968) suggest that the greater the complexity of a pattern in terms of its Fourier components, the weaker will be its effect upon channels "tuned" to those spatial frequencies. Accordingly, the effect of a square-wave grating would be more pronounced on the channel responding to that fundamental spatial frequency than would that of a single line having the same dimensions as a unit of the grating. The orientation selectivity of grating afterimages may then be a function of the relative sizes of the units activated, in the manner suggested above. This interpretation is, however, faced with certain difficulties. First, it has been demonstrated that the effect of a line on the visual system is similar to that of a grating with a spatial frequency of 5 cycles/deg (Bodis-Wollner, 1972; Sullivan, Georgeson, \& Oatley, 1972). Since orientation selectivity was clearly evident for afterimages of 4 cycles/deg, it would also have been expected for single-line afterimages, as these would have stimulated all frequencies, including $5 \mathrm{cycles} / \mathrm{deg}$, to which the visual system is most sensitive. That is, according to the above analysis, longer unitary durations would have been predicted for vertical than for $45-\mathrm{deg}$ line afterimages. An alternative hypothesis corresponds to one implied by Sullivan et al (1972), namely, that single lines are processed in the visual system differently from periodic patterns. Secondly, there were no significant effects of spatial frequency on afterimage duration in Experiment II. However, the contrast sensitivity functions do not show any marked differences in the range of 1.4 cycles/deg (Blakemore \& Campbell, 1969). Moreover, adaptation experiments with frequencies below 3 cycles/deg indicate that the threshold elevation occurs at around 3 cycles/deg rather than at the adapting spatial frequency (Blakemore \& Campbell, 1969). That is, frequencies below 3 cycles/deg appear to have the same effect on the visual system as those at around 3 cycles/deg. These points render the absence of afterimage duration effects in the range of 1.4 cycles/deg less of a problem.

In passing, it may be noteworthy that the stimulus most resistant to fragmentation during stabilization is a circle (Evans, 1965), which is, in Fourier terms, one of the most complex optical stimuli (Hopkins, 1962), as it contains a broad spectrum of spatial frequencies which are present in all orientations.

In conclusion, the orientation selectivity evident for grating but not for line afterimages appears to correspond to the selectivity in the EEG response to oscillating gratings.

\section{REFERENCES}

Atkinson, J. The effect of size, retinal locus, and orientation on the visibility of a single afterimage. Perception \& Fsychophysics. 1972. 12. 213-217.

Blakemore, $C$. \& Campbell. $\mathrm{F}$. W. On the existence of neurones in the human visual system selectively sensitive to the 
orientation and size of retinal images. Journal of Physiology. $1969,203,237-260$.

Blakemore, C.. Nachmias. J.. \& Sutton. P. The perceived spatial frequency shift: Evidence for frequency-selective neurones in the human brain. Journal of Physiology. 1970. 210. 727-750.

Bodis-Wollner, I. Contrast sensitivity and increment threshold. Perception, 1972. 1. 73-83.

Campbell, F. W., Cooper, G. F., \& Enroth-Cugell. C. The spatial selectivity of the visual cells of the cat. Journal of Physiology. 1969, 203, 223-235.

Campbell, F. W.. \& Maffei. L. Electrophysiological evidence for the existence of orientation and size detectors in the human visual system. Journal of Physiology, 1970, 207, 635-652.

Campbell, F. W., \& Robson. J. G. Application of Fourier analysis to the visibility of gratings. Journal of Physiology. 1968, 197. 551-566.

Craig. E. A., \& Lichtenstein, M. Visibility-invisibility cycles as a function of stimulus orientation. American Joumal of Psychology, 1953, 66. 554-563.

Evans, C. R. Some studies of pattern perception using a stabilized retinal image. British Journal of Psychology, 1965 $56,121-133$.

Evans, C. R. Further studies of pattern perception and a stabilized retinal image: The use of prolonged afterimages to achieve perfect stabilization. British Journal of Psychology, $1967,58,315-327$.

Hopkins, H. H. The application of frequency response techniques in optics. Proceedings of the Physical Society, 1962, 79, 889-919.

Howard, I. P., \& Templeton, W. B. Human spatial orientation. New York: Wiley, 1966

Maffei. L., \& Campbel. F. W. Neurophysiological localization of the vertical and horizontal visual coordinates in man. Science, $1970,167,386-387$.

Pantle, A.. \& Sekuler, R. Size-detecting mechanisms in human vision. Science, 1968, 162, 1146-1148.
Rodger, R. S. Internediate statistics. Sydney: University Cooperative Bookshop, 1965.

Schmidt, M. J., Fulgham, D. D., \& Brown, D. R. Stabilized images: The search for pattern elements. Perception \& Psychophysics, 1971, 10, 295-299.

Sullivan, G. D.. Georgeson, M. A., \& Oatley, K. Channels for spatial frequency selection and the detection of single bars by the human visual system. Vision Research, 1972, 12, 383-394.

Wade, N. J. Orientation effects on line afterimages. Perception \& Psychophysics, 1972, 12, 409-416.

Weisstein, N. What the frog's eye tells the human brain: Single cell analyzers in the human visual system. Psychological Bulletin, 1969, 72, 157-176.

\section{NOTES}

1. Data for each minute of observation showed the same relative effects, and thus the data over the total 3-min inspection period are given.

2. The observation interval of $60 \mathrm{sec}$ employed by Evans and Atkinson was arbitrary, and in fact quite different estimates of unit size would have been derived from using other intervals. From the results of the previous investigation (Wade, 1972), it can be seen that, in general, the longer the observation interval used, the larger will be the estimated size of the.functional units, as the duration of unitary relative to partial afterimage disappearance increases with increased observation. Therefore, the observation interval employed is of critical importance in such estimations, and must be taken into account in assessing the validity of this approach.

(Received for publication October 9, 1972; revision received January $17,1973$. 